\title{
Superior Mesenteric Vein Thrombosis in a Patient on Oral Contraceptive Pills
}

\author{
Prashanth Rawla ${ }^{a}$, e, Jeffrey Pradeep Raj ${ }^{b}$, Krishna Chaitanya Thandrac, \\ Sathayajit S. Bandaru ${ }^{\mathrm{d}}$
}

\begin{abstract}
Abdominal pain is a common occurrence, and it may vary from subtle pain to very severe pain due to a variety of causes, some of which may even be life-threatening. It is commonly believed that a life-threatening cause is almost always very severe with regards to the symptomatology. However, here in this case report, we report an unusual case of abdominal pain due to superior mesenteric vein (SMV) thrombosis in an otherwise healthy lady who was on an oral contraceptive agent for the last 2 years. She presented with mild abdominal pain for 3 days, and a computed tomography scan of the abdomen which was done to rule out common surgical causes of abdominal pain revealed a thrombus in the SMV. The patient was immediately started on systemic anticoagulation and was subsequently cured of her illness completely. This case report thus serves as a reminder to physicians that all abdominal pain should be thoroughly evaluated irrespective of the severity of symptoms.
\end{abstract}

Keywords: SMV; Thrombosis; OCP

\section{Introduction}

Abdominal pain is a common occurrence, and every human being experiences them often. The causes may be subtle like constipation, abdominal bloating or gastritis to something life-threatening like intestinal obstruction or perforation [1]. It is a logical understanding that many of us including health care physicians believe that anything sinister would be more severe and vice versa. However, in this case, we report a case of non-severe abdominal pain but due to a potentially danger-

Manuscript submitted November 3, 2017, accepted November 30, 2017

a Department of Internal Medicine, Memorial Hospital of Martinsville and Henry County, VA, USA

bDepartment of Pharmacology, St John's Medical College, Bangalore, India ${ }^{\mathrm{c}}$ Department of Critical Care Medicine, Memorial Sloan Kettering Cancer Center, New York, NY, USA

${ }^{\mathrm{d} B e t h}$ Israel Deaconess Medical Center, Harvard Medical School, Boston, MA, USA

'Corresponding Author: Prashanth Rawla, Department of Internal Medicine, Memorial Hospital of Martinsville and Henry County, VA, USA.

Email: rawlap@gmail.com

doi: https://doi.org/10.14740/gr903w ous etiology which if it was diagnosed late could have caused severe morbidity. This case report we believe would serve as a reminder to us doctors that we should be on the look-out for severe underlying etiology though the clinical presentation may be subtle with not much of a patient discomfort.

\section{Case Report}

A 38-year-old woman who was apparently well before 3 days presented with an insidious onset peri-umbilical pain. The pain was continuous, dull aching and was associated with intermittent nausea but there was no history of any vomiting or fever. On a pain rating scale out of 10 , the patient ranked the pain at 5. There was no recent change in bowel movement, and there were no known aggravating or relieving factors. She was currently not in her bleeding phase of her menstrual cycle. She has been a known hypertensive and suffered from hypothyroidism for the past 5 years. Her medication history included amlodipine $5 \mathrm{mg}$ once daily and levothyroxine $50 \mu \mathrm{g}$ once daily in the morning. She was an occasional consumer of alcohol but smokes cigarettes 0.5 pack a day for the past 10 years. At this stage, we considered to rule out any surgical causes of abdominal pain. A physical examination on admission demonstrated tenderness around the umbilical area with slight abdominal distention, but there was no rebound tenderness. There were also no signs suggestive of peritonitis. She was afebrile with normal vital signs. Her complete blood profile revealed a total white blood cell count of $7.2 \times 10^{3} / \mu \mathrm{L}$ with a differential count of 4,270 neutrophils $/ \mu \mathrm{L}(70 \%)$ and 927 lymphocytes $/ \mu \mathrm{L}(15 \%)$. Platelets were $235 \times 10^{3} / \mu \mathrm{L}$, and the hemoglobin levels were $12.6 \mathrm{~g} / \mathrm{dL}$. Liver function tests revealed alanine transaminase $43 \mathrm{UI} / \mathrm{L}$, aspartate transaminase $47 \mathrm{UI} / \mathrm{L}$ and alkaline phosphatase $142 \mathrm{IU} / \mathrm{L}$. The C-reactive protein level was $9 \mathrm{mg} / \mathrm{L}$. These findings ruled out the possibility of commonest surgical causes of abdominal pain like appendicitis, cholecystitis, and pancreatitis. However, to confirm, contrast-enhanced computed tomography (CECT) of the abdomen was performed. It revealed an intraluminal filling defect in the superior mesenteric vein (SMV) extending to the portal vein which was consistent with SMV thrombosis. The computed tomography (CT), however, revealed no signs of bowel ischemia and peritoneal fluid was absent (Fig. 1). A thorough history was asked to find out the cause of thrombosis. There was no history of any recent surgery, immobilization, or history of any previous thrombotic events. The patient then informed us that she has been 


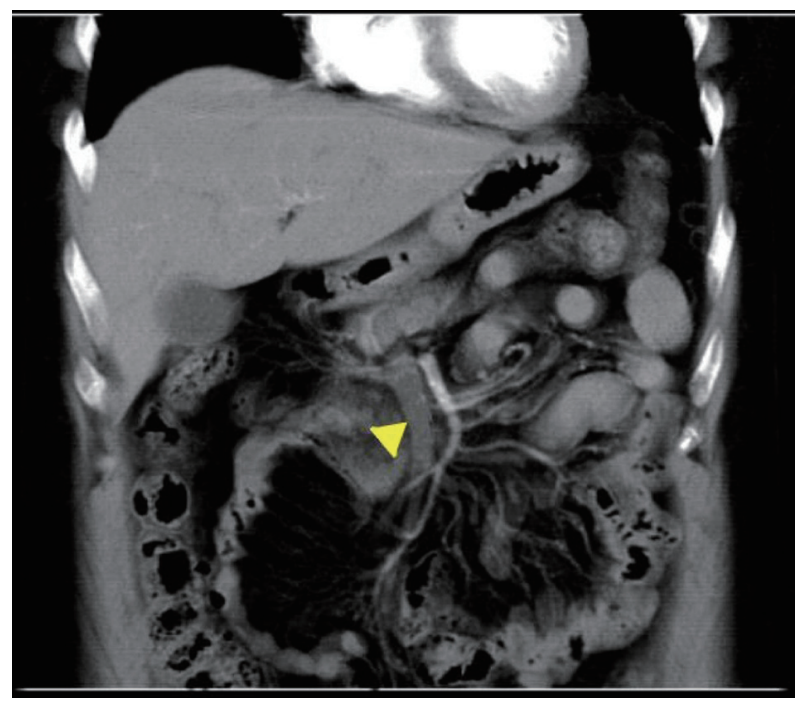

Figure 1. CT of the abdomen done at admission with intravenous contrast revealed thrombus in the SMV extending into the portal vein (yellow arrow head). CT: computed tomography; SMV: superior mesenteric vein.

taking a combined hormonal contraceptive containing $20 \mu \mathrm{g}$ ethinylestradiol and $0.1 \mathrm{mg}$ levonorgestrel for the last 2 years. Hence the diagnosis was confirmed as oral contraceptive pills (OCPs) induced SMV thrombosis. The patient was thus treated with weight-based heparin drip and monitored with serial abdominal exams. Her symptoms gradually subsided 2 days after the initiation of therapy, and heparin was slowly transitioned to warfarin. A CECT of the abdomen done 2 weeks later showed no evidence of any thrombus in the SMV. Follow-up examination for hypercoagulability demonstrated normal antithrombin III, protein $\mathrm{C}$, and protein $\mathrm{S}$ activity.

\section{Discussion}

Mesenteric vein thrombosis (MVT) which was earlier believed to be responsible for only a small minority of patients with intestinal ischemia which was previously recognized either at surgery or post-mortem is now thought to be increasing in incidence mainly due to the advent of newer diagnostics and advancement in radio-imaging technologies [2]. This statement is better proved by the findings of Acosta et al, wherein the incidence of MVT increased from 2.0 per 100,000 patient years between 1970 and 1982 to 2.7 per 100,000 patient years between the years 2000 and 2006 [3]. The onset of MVT may be acute, sub-acute or chronic. The pathophysiology of MVT lines up with the Virchow's classic triad of venous stasis, endothelial injury and hypercoagulability state. At least one of these three risk factors is said to be recognized in most patients [2]. In our patient, the risk factor identified is hypercoagulability state secondary to an intake of OCPs and smoking habit.

Combined OCPs are a well-known risk factor for venous thromboembolism (VTE). A study from the United Kingdom examining over 10,000 cases of venous thrombosis reported an increased risk of VTE of nearly three times when on cur- rent exposure to OCP. The risk is higher for first-generation progesterone containing OCPs than those containing the second generation ones. The number of cases of VTE per year per 10,000 treated women was lowest for levonorgestrel (six cases) when compared to desogestrel and cyproterone (14 cases) which are the highest [4]. About $9 \%$ of women of reproductive age worldwide use OCPs, and this number increases to $18 \%$ in developed regions [5]. The most typical sites of VTE while on OCP use are deep veins of the legs and then in the pulmonary vasculature. MVT related to OCP use accounts for just $4-5 \%$ of all MVTs [6]. The incidence of MVT itself being sporadic, OCP induced MVT is quite rare and could be picked up only with a high degree of suspicion.

As we are aware, the VTE is dependent on the estrogens component, and the newer generation progesterone exerts some protective effect [6]. The mechanisms responsible for the thromboembolic phenomena in OCP users are not very well defined. However there are several mechanisms which are postulated: 1) OCPs may induce a prothrombotic state by acceleration of both the extrinsic and intrinsic pathways of coagulation cascade or by decreasing the antithrombin III levels; 2) OCPs may cause anti-fibrinolytic activity by reducing spontaneous fibrinolysis; 3) they may induce intimal hyperplasia [7].

The clinical features of MVT depend on the site and timing of the thrombus formation. The thrombus formed in the large vein is less likely to cause intestinal infarction than the one formed in the peripheral mesenteric veins. Similarly, an acute onset MVT would produce a severe colicky mid-abdominal pain which is out-of-proportion to the clinical findings in the abdomen [8]. In our patient since the thrombus was in the larger SMV, there was less ischemic damage to the intestine, and thus the symptoms were not severe. There are also a few case reports and post-mortem series which suggest that SMV thrombosis does not cause intestinal infarction in nearly $50 \%$ of the patients [7] which is similar to our case scenario. The literature also suggests that the duration of symptoms before seeking medical attention typically ranges from an average of 5 - 14 days and nearly $75 \%$ of them reporting a minimum of 2 days of abdominal pain [9]. Our patient reported 3 days of pain at presentation, and she was rightly managed by early diagnosis using radio-imaging and appropriately treated with systemic anticoagulation.

According to the WHO causality assessment, OCP is the "possible" cause for SMV thrombosis as dechallenge was not possible to delineate the causality with clarity and rechallenge was not done. According to the Naranjo algorithm, the causality is again "possible" with a score of 2 [10]. According to the Schumock and Thornton preventability scale, the adverse drug reaction (ADR) is unpreventable [11] and based on the Hartwig and Siegel severity assessment scale, the severity of the reaction is placed at level 4 which involves requirement of another drug or treatment and the adverse effect being the cause of the admission with no need of stay in the intensive care unit [10].

\section{Conclusions}

Patients presenting with SMV thrombosis and using OCPs 
are rare. SMV thrombosis not causing intestinal ischemia or infarction is rare. It starts off like a non-specific abdominal pain, and if not diagnosed and treated early, it can lead to intestinal ischemia and gangrene formation which is associated with severe morbidity and high mortality. If not for the timely diagnosis and early management of SMV thrombosis in our patient with immediate systemic anticoagulant therapy, the consequences would have been serious which might range from intestinal infarction to even the death of the patient. This case report serves as a wake-up call for doctors to carefully evaluate all abdominal pains irrespective of the severity. SMV thrombosis should be looked for in patients on OCPs or who have other high-risk factors for thrombosis. Our experience also suggests that early diagnosis of the etiology of abdominal pain with use of available newer technology whenever affordable and feasible would go a long way in cutting down the morbidity and mortality.

\section{Conflict of Interest}

The authors do not have any conflict of interest to disclose.

\section{Financial Support}

This research received no specific grant from any funding agency in the public, commercial, or not-for-profit sectors.

\section{Author Contributions}

Study design by PR. Drafting, critical revisions and final approval by PR, JPR, KCT, and SSB.

\section{References}

1. Macaluso CR, McNamara RM. Evaluation and management of acute abdominal pain in the emergency department. Int J Gen Med. 2012;5:789-797.

2. Harnik IG, Brandt LJ. Mesenteric venous thrombosis. Vasc Med. 2010;15(5):407-418.

3. Acosta S, Alhadad A, Svensson P, Ekberg O. Epidemiology, risk and prognostic factors in mesenteric venous thrombosis. Br J Surg. 2008;95(10):1245-1251.

4. Vinogradova Y, Coupland C, Hippisley-Cox J. Use of combined oral contraceptives and risk of venous thromboembolism: nested case-control studies using the QResearch and CPRD databases. BMJ. 2015;350:h2135.

5. Department of Economic and Social Affairs. World contraceptive patterns of 2013 United Nations, 2013.

6. Hassan HA. Oral contraceptive-induced mesenteric venous thrombosis with resultant intestinal ischemia. J Clin Gastroenterol. 1999;29(1):90-95.

7. Miller DR. Unusual focal mesenteric venous thrombosis associated with contraceptive medication: a case report. Ann Surg. 1971;173(1):135-138.

8. Brandt LJ, Boley SJ. AGA technical review on intestinal ischemia. American Gastrointestinal Association. Gastroenterology. 2000;118(5):954-968.

9. Orr DW, Harrison PM, Devlin J, Karani JB, Kane PA, Heaton ND, O'Grady JG, et al. Chronic mesenteric venous thrombosis: evaluation and determinants of survival during long-term follow-up. Clin Gastroenterol Hepatol. 2007;5(1):80-86.

10. Srinivasan R, Ramya G. Adverse drug reaction-causality assessment. Int J Res Pharm Chem. 2011;1(3):606-612.

11. Schumock GT, Thornton JP. Focusing on the preventability of adverse drug reactions. Hosp Pharm. 1992;27(6):538. 